\title{
PAPER
}

\section{New evidence for an association between the CSF HVA:5-HIAA ratio and psychopathic traits}

\author{
H Soderstrom, K Blennow, A-K Sjodin, A Forsman
}

J Neurol Neurosurg Psychiatry 2003;74:918-921

See end of article for
authors' affiliations
$\ldots \ldots \ldots \ldots \ldots \ldots \ldots \ldots \ldots$
Correspondence to:
Dr H Soderstrom,
Department of Forensic
Psychiatry, Box 4024, 422
04 Hisings Backa,
Sweden;
henrik.soderstrom@rmv.se
Received 31 May 2002
In revised form
8 November 2002
Accepted
13 December 2002

\begin{abstract}
Objectives: To replicate the relation between the CSF HVA:5-HIAA ratio and psychopathic traits previously reported in a pilot group of 22 perpetrators of violent crimes.

Methods: CSF monoamine metabolite concentrations in another 28 violent and sexual offenders, aged 45 or below, referred to pretrial forensic psychiatric investigation, were compared to features of psychopathy according to the Psychopathy Checklist-Revised (PCL-R).

Results: Our previous finding was repeated in the new study group, where the HVA:5-HIAA ratio was strongly associated with psychopathic traits $(r=0.50, p=0.010)$, particularly its behavioural aspects $(r=0.523, p=0.004)$. In subsamples of individuals from both study groups who had no medication $(n=25)$ or no current axis I disorder, including a history of mood disorder or substance dependence $(n=21)$, the HVA:5-HIAA ratio remained strongly associated with all psychopathy factors but most closely with the behavioural features. Retrospective assessments of childhood disruptive symptomatology, such as attention deficit hyperactivity disorder or conduct disorder, analysed in relation to the monoamine metabolites, showed the same association with the HVA:5-HIAA ratio.

Conclusions: Violent and aggressive behavioural traits with childhood onset and adult expression as psychopathic features are associated with changed activity in the brain dopaminergic system, possibly as a result of serotonergic dysregulation.
\end{abstract}

$\mathrm{P}$ sychopathy is the personality profile most closely related to violent and dangerous aggression. It is characterised by inconsistent, impulsive behaviour and distorted perceptions of interpersonal and temporal processes with a propensity to violate the rights of others in order to achieve dominance. ${ }^{1}$ Similar behaviours in childhood are included in the diagnostic criteria for attention deficit-hyperactivity disorder (ADHD) and conduct disorder (CD). Whereas low cerebrospinal fluid (CSF) 5-hydroxyindoleacetic acid (5HIAA) concentrations have been a consistent finding in self destructively aggressive patients ${ }^{2-5}$ and a relation between low 5-HIAA and aggressive behaviour has been reported in depressive, personality disordered, or highly impulsive individuals, ${ }^{6-8}$ low 5-HIAA concentrations have not explained or predicted outward directed, non-impulsive violent behaviour in non-disordered subjects. ${ }^{4}$ The ratio between the metabolites of serotonin and dopamine is highly constant, ${ }^{9}$ and an increased homovanillic acid (HVA):5-HIAA ratio indicates an impaired serotonergic modulation of dopamine activity. ${ }^{10}$

To assess the traits linked to psychopathy, Hare and coworkers $^{11}$ developed a 20 item clinical and actuarial rating scale, the Psychopathy Checklist-Revised (PCL-R). Ratings yield a two factor structure, where interpersonal/affective aspects (item $1=$ glibness/superficial charm, item $2=$ grandiose sense of self worth, item $4=$ pathological lying, item $5=$ conning/manipulative, item $6=$ lack of remorse or guilt, item $7=$ shallow affect, item $8=$ callous/lack of empathy, and item $16=$ failure to accept responsibility for own actions) are reflected by factor 1 and social behavioural deviance (item 3 = need for stimulation/proneness to boredom, item $9=$ parasitic lifestyle, item $10=$ poor behavioural control, item 12 = early behavioural problems, item $13=$ lack of realistic, long term goals, item $14=$ impulsivity, item $15=$ irresponsibility, item $18=$ juvenile delinquency, item $19=$ revocation of conditional release) by factor $2 .^{12}{ }^{13}$ Three of 20 items (item $11=$ promiscuous sexual behaviour, item $17=$ many short term marital relationships, item $20=$ criminal versatility) did not load on either factor. A recently published three factor model ${ }^{14}$ excludes items directly describing criminal activities and divides factor 1 into "deceitful interactive style" (new factor 1, including items 1, 2, 4, and 5) "affective hyporesponsiveness" (new factor 2, including items 6, 7, 8 , and 16), and "impulsive and inconsistent behaviour" (new factor 3, including items 3, 9, 13, 14, and 15). The total and factor PCL-R scores correlate linearly or semilinearly with criminal behaviour ${ }^{15}{ }^{16}$ and with dimensional ratings of axes I and II DSM disorders. ${ }^{17}$

In a previous study, ${ }^{18}$ we found a close correlation between the CSF HVA:5-HIAA ratio and all aspects of psychopathy among 22 violent offenders (aged 17-67 years) referred for pretrial forensic psychiatric investigation. This finding was not explained by age, axis 1 disorders, including a history of substance abuse/dependence, or current medication. We have now repeated this study in a new sample of offenders to test again for covariance between CSF metabolite concentrations and psychopathic traits, and also included careful retrospective diagnoses of childhood disruptive disorders in the analyses to further characterise the clinical correlates to the HVA:5-HIAA ratio among offenders.

\section{PATIENTS AND METHODS Study population}

All subjects of inpatient forensic psychiatric investigations in Gothenburg from October 1998 to February 2001 who were charged with a severe crime (homicide, attempted homicide, aggravated assault, arson, rape, or sexual violation of minors) and had received a basic Swedish education were eligible for participation in the "Gothenburg Forensic Neuropsychiatry

Abbreviations: 5-HIAA, 5-hydroxyindoleacetic acid; ADHD, attention deficit-hyperactivity disorder; $C D$, conduct disorder; CSF, cerebrospinal fluid; HVA, homovanillic acid; MHPG, 3-methoxy-4-hydroxyphenylglycol; PCL-R, Psychopathy Checklist-Revised 
Table 1 Correlations (Spearman's rank test) between the monoamine metabolites and PCL-R scores

\begin{tabular}{|c|c|c|c|c|c|}
\hline & \multirow[b]{2}{*}{$\begin{array}{l}\text { New sample } \\
(n=28)\end{array}$} & \multicolumn{4}{|l|}{ Pooled samples } \\
\hline & & $\begin{array}{l}\text { All subjects } \leqslant 45 \\
\text { years }(n=47)\end{array}$ & $\begin{array}{l}\text { All subjects without } \\
\text { medication }(n=25)\end{array}$ & $\begin{array}{l}\text { All subje } \\
\text { axis I dia }\end{array}$ & $\begin{array}{l}\text { ects without } \\
\text { agnosis }(n=21)\end{array}$ \\
\hline \multicolumn{6}{|l|}{ HVA } \\
\hline PCL-R: total score & $0.32(p=0.092)$ & $0.36(p=0.014)$ & $0.51(p=0.010)$ & 0.42 & $(p=0.056)$ \\
\hline Factor 1 & $0.02(p=0.93)$ & $0.15(p=0.33)$ & $0.20(p=0.35)$ & 0.28 & $(p=0.22)$ \\
\hline Factor 2 & $0.39(p=0.038)$ & $0.50(p<0.001)$ & $0.66(p<0.001)$ & 0.59 & $(p=0.005)$ \\
\hline New factor 1 & $0.33(p=0.081)$ & $0.26(p=0.075)$ & $0.25(p=0.22)$ & 0.18 & $(p=0.44)$ \\
\hline New factor 2 & $-0.03(p=0.89)$ & $0.22(p=0.15)$ & $0.40(p=0.049)$ & 0.33 & $(p=0.15)$ \\
\hline New factor 3 & $0.33(p=0.086)$ & $0.35(p=0.016)$ & $0.40(p=0.047)$ & 0.42 & $(p=0.055)$ \\
\hline \multicolumn{6}{|l|}{ 5-HIAA } \\
\hline PCL-R: total score & $0.02(p=0.92)$ & $0.08(p=0.60)$ & $0.20(p=0.35)$ & 0.06 & $(p=0.80)$ \\
\hline Factor 1 & $-0.17(p=0.40)$ & $-0.07(p=0.66)$ & $-0.07(p=0.73)$ & -0.04 & $(p=0.87)$ \\
\hline Factor 2 & $0.07(p=0.72)$ & $0.22(p=0.14)$ & $0.33(p=0.10)$ & 0.26 & $(p=0.26)$ \\
\hline New factor 1 & $0.17(p=0.40)$ & $0.07(p=0.63)$ & $0.03(p=0.88)$ & -0.13 & $(p=0.56)$ \\
\hline New factor 2 & $-0.22(p=0.27)$ & $-0.03(p=0.86)$ & $0.15(p=0.48)$ & 0.11 & $(p=0.65)$ \\
\hline New factor 3 & $-0.01(p=0.98)$ & $0.09(p=0.54)$ & $0.11(p=0.61)$ & 0.07 & $(p=0.77)$ \\
\hline \multicolumn{6}{|l|}{ MHPG } \\
\hline PCL-R: total score & $0.30(p=0.136)$ & $0.38(p=0.009)$ & $0.45(p=0.023)$ & 0.55 & $(p=0.010)$ \\
\hline Factor 1 & $0.13(p=0.52)$ & $0.22(p=0.14)$ & $0.27(p=0.20)$ & 0.41 & $(p=0.065)$ \\
\hline Factor 2 & $0.26(p=0.18)$ & $0.46(p<0.001)$ & $0.51(p=0.010)$ & 0.60 & $(p=0.004)$ \\
\hline New factor 1 & $0.43(p=0.023)$ & $0.32(p=0.027)$ & $0.35(p=0.08)$ & 0.28 & $(p=0.21)$ \\
\hline New factor 2 & $0.10(p=0.63)$ & $0.28(p=0.059)$ & $0.39(p=0.056)$ & 0.37 & $(p=0.10)$ \\
\hline New factor 3 & $0.45(p=0.017)$ & $0.47(p<0.001)$ & $0.42(p=0.037)$ & 0.52 & $(p=0.015)$ \\
\hline \multicolumn{6}{|l|}{ HVA:5-HIAA } \\
\hline PCL-R: total score & $0.50(p=0.007)$ & $0.50(p<0.001)$ & $0.67(p<0.001)$ & 0.59 & $(p=0.005)$ \\
\hline Factor 1 & $0.28(p=0.16)$ & $0.35(p=0.016)$ & $0.39(p=0.052)$ & 0.51 & $(p=0.018)$ \\
\hline Factor 2 & $0.53(p=0.004)$ & $0.49(p<0.001)$ & $0.71(p<0.001)$ & 0.58 & $(p=0.006)$ \\
\hline New factor 1 & $0.40(p=0.037)$ & $0.40(p=0.005)$ & $0.36(p=0.081)$ & 0.45 & $(p=0.039)$ \\
\hline New factor 2 & $0.26(p=0.18)$ & $0.40(p=0.006)$ & $0.51(p=0.010)$ & 0.45 & $(p=0.043)$ \\
\hline New factor 3 & $0.49(p=0.008)$ & $0.42(p=0.003)$ & $0.55(p=0.004)$ & 0.53 & $(p=0.014)$ \\
\hline \multicolumn{6}{|l|}{ HVA:MHPG } \\
\hline PCL-R: total score & $0.11(p=0.57)$ & $0.50(p<0.001)$ & $0.23(p=0.28)$ & -0.01 & $(p=0.98)$ \\
\hline Factor 1 & $-0.07(p=0.74)$ & $0.35(p=0.016)$ & $0.03(p=0.87)$ & -0.11 & $(p=0.65)$ \\
\hline Factor 2 & $0.23(p=0.23)$ & $0.49(p<0.001)$ & $0.39(p=0.055)$ & 0.22 & $(p=0.35)$ \\
\hline New factor 1 & $0.13(p=0.53)$ & $0.40(p=0.005)$ & $0.05(p=0.80)$ & -0.11 & $(p=0.63)$ \\
\hline New factor 2 & $-0.11(p=0.58)$ & $0.40(p=0.006)$ & $0.17(p=0.43)$ & $-0.001 i$ & $(p=0.98)$ \\
\hline New factor 3 & $0.05(p=0.80)$ & $0.42(p=0.003)$ & $0.15(p=0.48)$ & 0.07 & $(p=0.77)$ \\
\hline \multicolumn{6}{|l|}{ 5-HIAA:MHPG } \\
\hline PCL-R: total score & $-0.20(p=0.31)$ & $-0.16(p=0.29)$ & $-0.07(p=0.74)$ & -0.28 & $(p=0.22)$ \\
\hline Factor 1 & $-0.23(p=0.25)$ & $-0.19(p=0.20)$ & $-0.18(p=0.39)$ & -0.35 & $(p=0.12)$ \\
\hline Factor 2 & $-0.07(p=0.73)$ & $-0.03(p=0.84)$ & $0.08(p=0.71)$ & -0.08 & $(p=0.75)$ \\
\hline New factor 1 & $-0.11(p=0.58)$ & $-0.14(p=0.35)$ & $-0.11(p=0.59)$ & -0.35 & $(p=0.12)$ \\
\hline New factor 2 & $-0.28(p=0.15)$ & $-0.18(p=0.24)$ & $-0.09(p=0.67)$ & -0.19 & $(p=0.41)$ \\
\hline New factor 3 & $-0.28(p=0.16)$ & $-0.20(p=0.18)$ & $-0.11(p=0.60)$ & -0.19 & $(p=0.42)$ \\
\hline
\end{tabular}

Project" aimed at evaluating neuropsychiatric dysfunctions in violent offenders. All potential subjects were given written information about the study and could choose to participate in some but not in other parts of the investigation. The project was approved by the Research Ethics Committee at Gothenburg University, Sweden.

A total of 121 subjects met the inclusion criteria; 100 consented to participate in some or all parts of the study. To have a homogeneous sample with regard to CNS function, we chose to study the subgroup aged $\leqslant 45$ years ( 69 men and 7 women, aged $17-45$, median 26.5 years). Twenty eight of these subjects (27 men and one woman, aged 18-45, median 29.5 years), accepted lumbar puncture. These subjects did not differ significantly from the whole study cohort $\leqslant 45$ years with respect to age $(p=0.08)$, gender $(p=0.20)$, medication $(p=0.27)$, violent crimes $(p=0.60)$, severe mental disorder $(\mathrm{p}=0.26)$, or PCL-R total scores $(\mathrm{p}=0.54)$ according to Sprearman's rank tests or Mann-Whitney U tests. Psychiatric diagnoses according to DSM-IV criteria were made on the basis of SCID-I interviews and all available file and clinical information. Special efforts were made to ascertain the true extent of all kinds of substance abuse in the history by means of collateral interviews and register information.

\section{Diagnoses}

Ten subjects (36\%) had no axis I disorder. The most common diagnosis was substance abuse, registered in 14 subjects $(50 \%)$, followed by mood disorder in nine $(32 \%)$, anxiety disorder in seven $(25 \%)$, and psychotic disorder in four subjects ( $14 \%)$. Ten subjects (36\%) had at some time attempted suicide.

\section{Medication}

All subjects with substance abuse or dependence had been detoxified while on remand before admission to the study department and had consumed no alcohol or non-prescribed drugs for at least one month. Fourteen subjects $(50 \%)$ were on no medication, four ( $14 \%$ ) were on antidepressants, two (7\%) were on minor tranquillisers, two (7\%) were on antiepileptics/ lithium, and five ( $18 \%$ ) were on more than one type of drug.

\section{Crimes}

The subjects were indicted for and subsequently convicted of the following crimes: murder/manslaughter in four cases (14\%), attempted murder/manslaughter in four (14\%), aggravated assault in four (14\%), sexual crimes against adults in four (14\%) sexual crimes against minors in nine $(32 \%)$, unlawful threats/coercion in two (7\%), and arson in one $(3 \%)$. 


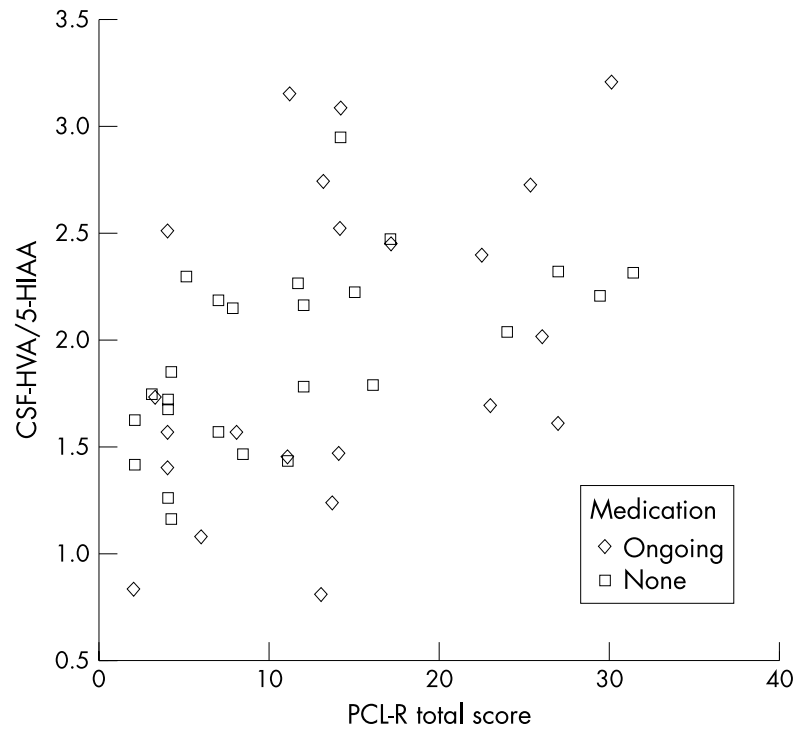

Figure 1 Correlation between PCL-R total score and the HVA:5-HIAA ratio.

\section{Rating procedures}

Each subject was rated on the PCL-R by a specially trained social worker. PCL-R factor scores were calculated as suggested by Hare and colleagues ${ }^{11}$ and by Cooke and Michie. ${ }^{14}$ To allow comparisons, both the two and the three factor PCL-R models were used in the analyses. The childhood onset disruptive behaviours were assessed in a clinical interview covering each DSM-IV ADHD and CD criterion during childhood. This information was compared to data from extensive school, social, and health care files, to parent interviews performed whenever possible, and to self rated symptomatology according to the Wender Utah Rating Scale. ${ }^{19}$ All raters were blind to the laboratory findings.

\section{CSF sampling and analysis}

Lumbar puncture was performed between 8 and 9 am under standard conditions in the L3/L4 or L4/L5 interspace and with the subject lying down and fasting since midnight. The first 12 $\mathrm{ml}$ CSF was collected in polypropylene tubes and gently mixed to avoid gradient effects. No CSF samples contained more than 500 erythrocytes per $\mu \mathrm{l}$. The CSF samples were centrifuged at $2000 \mathrm{~g}$ for 10 minutes to eliminate cells and other insoluble material. Aliquots were then stored at $-80^{\circ} \mathrm{C}$ until biochemical analysis.

Determination of the monoamine metabolites was performed by means of high performance liquid chromatography (HPLC) with electrochemical detection, as described by Blennow and coworkers. ${ }^{20}$

\section{Statistical analyses}

All statistics were calculated with the SPSS 10.0 software, using two tailed $\mathrm{p}$ values. The monoamine metabolite concentrations and the PCL-R scores were approximately normally distributed, but all associations were analysed by means of non-parametric Spearman rank correlations. Correlations between the monoamine metabolites and the PCL factors were first analysed separately in the new study group $(n=28)$, then in the total number of subjects investigated in the pilot and the replication studies who were 45 years or younger (thus adding 19 subjects from the previously studied sample to the new study group, yielding a total number of 47 subjects), and finally in the subsamples of subjects who had no medication $(n=25 / 47)$ or mood, psychotic, anxiety, or substance abuse disorder $(n=21 / 47)$. The combination of the two study groups yielded large enough subgroups without these possible confounding factors to permit separate statistical analyses of the relations between the monoamine metabolites and the PCL-R. The impact of current medication on the monoamine metabolites was also tested by comparison of the metabolite concentrations between the groups using $t$ tests. Correlation coefficients are given with 2 decimals, $\mathrm{p}$ values $>0.10$ with 2 decimals, and p values $<0.10$ with 3 decimals.

\section{RESULTS}

The total PCL-R scores ranged from 2 to 27 points, with a median of 9.7; the factor 1 scores from 1 to 11 (median 4); and the factor 2 scores from 0 to 16 (median 5 ). The factors of the three factor model ranged from 0 to 4 (median 0.5 ) for the new factor 1; from 0 to 8 (median 3 ) for the new factor 2; and from 0 to 8 (median 2.5) for factor 3.

The ranges of HVA (42-400, mean $186.3 \mathrm{nmol} / \mathrm{l}), 5$-HIAA (34-178, mean 97.3 nmol/1), and 3-methoxy-4hydroxyphenylglycol (MHPG) (18-69, mean $39.4 \mathrm{nmol} / \mathrm{l})$ were lower than those reported in normal controls, ${ }^{19}$ but differences in the sampling procedures (such as no overnight rest in the controls) complicate comparisons. There was a strong intercorrelation between HVA and 5-HIAA $(r=0.73$, $\mathrm{p}<0.001)$, but not between HVA and MHPG $(r=0.29$, $\mathrm{p}=0.13)$ or between MHPG and 5-HIAA $(r=0.13, \mathrm{p}=0.50)$.

Table 1 presents correlations between the CSF metabolite concentrations and the PCL-R factors, for the new sample $(n=28)$, for the total number of subjects $<45$ years of age drawn from both samples $(n=28+19=47)$, for the total number of subjects without medication from both samples $(\mathrm{n}=25)$, and for subjects without any diagnosis of a mood disorder, psychotic disorder, anxiety disorder, or lifetime substance abuse $(n=21)$. Figure 1 illustrates the relation between total PCL-R scores and the HVA:5-HIAA ratio in the total number of subjects.

The HVA:5-HIAA ratio also correlated with other measures of childhood onset disruptive disorders-that is, the DSM-IV criteria for conduct disorder (CD) and each of the symptom clusters of ADHD (table 2).

Table 2 Correlations (Spearman's r) between monoamine measures and ratings of disruptive behaviour symptoms in childhood (subjects from the new sample only)

\begin{tabular}{lllllll}
\hline & HVA & 5-HIAA & MHPG & HVA:5-HIAA & HVA:MHPG & 5-HIAA:MHPG \\
\hline Conduct disorder $(\mathrm{n}=28)$ & 0.24 & -0.05 & -0.04 & 0.50 & 0.23 & -0.01 \\
& $\mathrm{p}=0.21$ & $\mathrm{p}=0.81$ & $\mathrm{p}=0.86$ & $\mathrm{p}=0.007$ & $\mathrm{p}=0.25$ & $\mathrm{p}=0.65$ \\
Attention deficit $(\mathrm{n}=27)$ & 0.27 & 0.09 & 0.09 & 0.38 & 0.29 & 0.07 \\
& $\mathrm{p}=0.18$ & $\mathrm{p}=0.67$ & $\mathrm{p}=0.65$ & $\mathrm{p}=0.048$ & $\mathrm{p}=0.14$ & $\mathrm{p}=0.70$ \\
Hyperactivity disorder $(\mathrm{n}=27)$ & 0.31 & 0.02 & 0.26 & 0.48 & 0.23 & -0.09 \\
& $\mathrm{p}=0.12$ & $\mathrm{p}=0.91$ & $\mathrm{p}=0.20$ & $\mathrm{p}=0.01$ & $\mathrm{p}=0.25$ & $\mathrm{p}=0.65$ \\
\hline
\end{tabular}


Five subjects had attempted suicide by passive and five by violent means. As expected, the subjects with a history of violent or non-violent suicide attempts had lower 5-HIAA and HVA than subjects without prior suicide attempts (mean 5-HIAA 79.4 and $97.0 v 102.3 \mathrm{nmol} / \mathrm{l}$; and mean HVA 149.0 and 155.3 v $205.1 \mathrm{nmol} / \mathrm{l})$.

The $t$ tests for differences in monoamine metabolite concentrations between medicated $(n=14)$ and unmedicated $(\mathrm{n}=14)$ subjects showed no significant differences (HVA: $t=-0.10, \quad \mathrm{df}=26, \mathrm{p}=0.92 ; 5$-HIAA: $t=-0.26, \mathrm{df}=26$, $\mathrm{p}=0.79$; MHPG: $t=0.74, \mathrm{df}=26, \mathrm{p}=0.46)$. In this study group, there were no significant correlations between age and monoamine metabolites (HVA: $r=-0.18, \mathrm{p}=0.36$; 5-HIAA: $r=-0.08, \mathrm{p}=0.68$; MHPG: $r=-0.19, \mathrm{p}=0.34)$.

\section{DISCUSSION}

The PCL-R scores were consistently low in our samples as they have been in previous Swedish samples. Since the PCL-R shows a consistent relation with violence, recidivism, and mental disorder, ${ }^{15-17}$ we think it may be accurate to interpret associations between biological measures and PCL-R scores in terms of psychopathic traits as opposed to categorical diagnoses of psychopathy versus non-psychopathy.

We chose not to exclude subjects with medication that may influence monoamine metabolite concentrations as this would have biased the samples, and since termination of medication may result in changes in the metabolite pattern even after a prolonged washout period..$^{21}$ The groups with or without medication did not differ in monoamine metabolite concentrations, and findings in the two independent samples remained virtually unchanged when separately analysed in the subgroup without medication or among the subjects without a lifetime diagnosis of a mood, psychotic, anxiety, or substance abuse disorder.

The HVA:5-HIAA ratio was thus significantly associated with psychopathic traits in two independent samples, collected consecutively in subsequent time periods. The fact that the ratio between the transmitter metabolites is more stable than the absolute concentrations of the individual metabolites supports the interpretation of the finding as a true biological characteristic associated with the clinically rated traits, most clearly with the outward directed aggressive and disorganised behaviour features, such as need for stimulation, poor behavioural control, impulsivity, and irresponsibility reflected by the PCL-R factor 2 and new factor 3 . The same association was revealed by retrospective assessments of childhood onset disruptive behaviours, which is compatible with previous findings in disruptive children, ${ }^{22}$ and suggests that psychopathic traits might be an adult sequel to ADHD and CD. Though childhood conditions can rarely be retrospectively diagnosed in adults with absolute certainty, we used a wide range of information from several independent sources to rate the number of symptoms fulfilled during childhood. Prospective longitudinal studies are required to provide firm data on how childhood behavioural traits develop into adulthood psychosocial dysfunction, but until such data are available, retrospective information must be cautiously used to trace developmental lines expressed in adult symptomatology.

Our findings permit the assumption that aggression might be related to a high dopamine turnover (possibly as an adaptation to post-synaptic dysfunction or due to a deficient serotonergic tonic regulation of the dopamine system) in combination with a relative serotonergic dysregulation (leading to disinhibition of aggressive impulses). Given this background, dopamine modulating drugs, alone or in combination with serotonin reuptake inhibitors (or drugs with combined dopamine and serotonin modulating action), might be of interest in treatment of aggressive psychopathy.

\section{ACKNOWLEDGEMENTS}

Agneta Brimse and Monika Montell are gratefully acknowledged for their skills with data and words.

\section{Authors' affiliations}

H Soderstrom, A-K Sjodin, Institute of Clinical Neuroscience,

Department of Psychiatry, Gothenburg University, and Department of Forensic Psychiatry, Gothenburg, Sweden

K Blennow, Institute of Clinical Neuroscience, Department of Neurology and Neurochemistry, Gothenburg University, Sweden

\section{REFERENCES}

1 Cleckley H. The mask of sanity, 1st edn. St Louis, MO: Mosby, 1941

2 Asberg M, Träskman L, Thorén P. 5-HIAA in the cerebrospinal fluid: a biochemical suicide predictor? Arch Gen Psychiatry 1976;33:1 193-7.

3 Lidberg L, Asberg M, Sundqvist-Stensman UB. 5-hydroxyindoleacetic acid levels in attempted suicides who have killed their children. Lancet 1984;ii:928.

4 Lidberg L, Tuck JR, Asberg M, et al. Homicide, suicide and CSF 5-HIAA. Acta Psychiatr Scand 1985;71:230-6.

5 Virkkunen M, DeJong J, Bartko J, et al. Psychobiological concomitants of history of suicide attempts among violent offenders and impulsive fire setters. Arch Gen Psychiatry 1989;46:604-6.

6 Brown GL, Goodwin FK, Ballenger JC, et al. Aggression in humans correlates with cerebrospinal fluid amine metabolites. Psychiatry Res 1979;1:131-9

7 Linnoila $M$, Virkkunen $M$, Scheinin $M$, et al. Low cerebrospinal fluid 5-hydroxyindoleacetic acid concentration differentiates impulsive from nonimpulsive violent behavior. Life Sci 1983:33:2609-14.

8 Stanley B, Molcho A, Stanley M, et al. Association of aggressive behavior with altered serotonergic function in patients who are no suicidal. Am J Psychiatry 2000;157:609-14.

9 Geracioti TD, Keck PE, Ekhator NN, et al. Continuous covariability of dopamine and serotonin metabolites in human cerebrospinal fluid. Biol Psychiatry 1008;44:228-33

10 Agren H, Mefford IN, Rudorfer MV, et al. Interacting neurotransmitter systems. a non-experimental approach to the 5-HIAA/HVA correlation in human CSF. J Psychiatry Res 1986;20:175-93.

11 Hare RD, Harpur TJ, Hakstian AR, et al. The revised psychopathy checklist: reliability and factor structure. Psychol Assessm J Consult Clin Psychol 1990;2:338-41

12 Harpur TJ, Hakstian AR, Hare RD. Factor structure of the Psychopathy Checklist. J Consult Clin Psychol 1988:56:741-7.

13 Harpur TJ, Hare RD, Hakstian AR. Two-factor conceptualization of psychopathy: construct validity and assessment implications. Psychol Assessm 1989;1:6-17.

14 Cooke DJ, Michie C. Refining the construct of psychopathy: towards a hierarchical model. Psychol Assessm 2001;13:171-88.

15 Hart SD. The role of psychopathy in assessing risk for violence. Conceptual and methodological issues. Leg Crim Psychol 1998;3: 123-40.

16 Hemphill J, Hare RD, Wong S. Psychopathy and recidivism. A review. Leg Crim Psychol 1998;3:141-72.

17 Hart SD, Hare RD. Discriminant validity of the psychopathy checklist in a forensic psychiatric population. Psychol Assessm 1989;1:211-18.

18 Soderstrom H, Blennow K, Manhem A, et al. CSF studies in violent offenders. I. 5-HIAA as a negative and HVA as a positive predictor of psychopathy. J Neural Transm 2001;108:869-78.

19 Ward MF, Wender PH, Reimherr FW. The Wender Utah Rating Scale: an aid in the retrospective diagnosis of childhood attention deficit hyperactivity disorder. Am J Psychiatry 1993;150:885-90.

20 Blennow K, Wallin A, Gotffries CG, et al. Cerebrospinal fluid monoamine metabolites in 114 healthy individuals, 18-88 years of age. Eur Neuropsychopharmacol 1993;3:55-61

21 Harnryd C, Bierkenstedt L, Gullberg B, et al. Time course for effects of sulpiride and chlorpromazine on monoamine metabolites and prolactin levels in cerebrospinal fluid from schizophrenic patients. Acta Psychiatr Scand 1984;311(suppl):75-92.

22 Castellanos FX, Elia J, Kruesi M, et al. Cerebrospinal fluid monoamine metabolites in boys with attention-deficit hyperactivity disorder. Psychiatry Res 1994;52:305-16. 